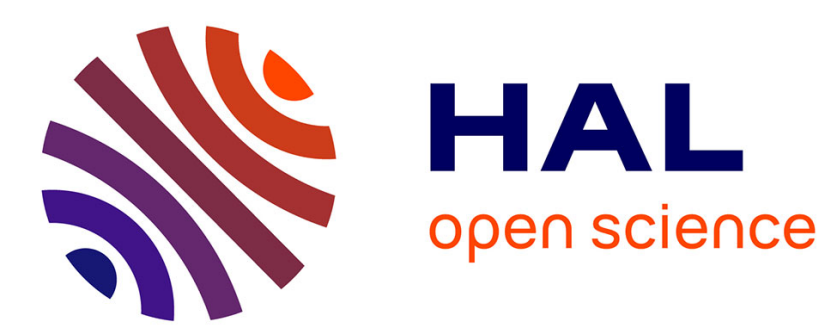

\title{
Matériaux pour guides de lumière
}

\author{
H.A. Combet
}

\section{To cite this version:}

H.A. Combet. Matériaux pour guides de lumière. Revue de Physique Appliquée, 1975, 10 (6), pp.349353. 10.1051/rphysap:01975001006034900 . jpa-00243925

\section{HAL Id: jpa-00243925 https://hal.science/jpa-00243925}

Submitted on 1 Jan 1975

HAL is a multi-disciplinary open access archive for the deposit and dissemination of scientific research documents, whether they are published or not. The documents may come from teaching and research institutions in France or abroad, or from public or private research centers.
L'archive ouverte pluridisciplinaire HAL, est destinée au dépôt et à la diffusion de documents scientifiques de niveau recherche, publiés ou non, émanant des établissements d'enseignement et de recherche français ou étrangers, des laboratoires publics ou privés. 


\title{
MATÉRIAUX POUR GUIDES DE LUMIÈRE
}

\author{
H. A. COMBET \\ Centre National d'Etudes des Télécommunications \\ Groupement C. P. M., 22301 Lannion, France
}

\begin{abstract}
Résumé. - Cette note est une synthèse bibliographique des problèmes liés à la préparation et à la caractérisation des matériaux ayant les propriétés désirées pour la réalisation des nouveaux systèmes de télécommunications optiques.

Abstract. - This paper is a review of the problems associated with the synthesis and characterization of materials having the wanted properties in view of their application to new optical telecommunications systems.
\end{abstract}

1. Introduction. - L'augmentation actuelle du trafic des télécommunications laisse entrevoir à terme la saturation des artères de transmission basées sur les techniques classiques: paires bifilaires, "câbles coaxiaux, etc... Face à l'énorme augmentation de bande passante a priori prévisible grâce à l'emploi de fréquences plus élevées, il n'est pas étonnant de voir se développer d'importantes recherches sur les télécommunications optiques dans les différents organismes s'intéressant aux télécommunications. Or le développement de ces nouveaux systèmes est particulièrement tributaire de progrès nécessaires dans le domaine de la science des matériaux.

Le but de cette note est donc de faire le point sur les problèmes liés à la synthèse, à la mise en forme, et à la caractérisation des matériaux ayant les propriétés voulues pour ces applications, en soulignant les progrès qui restent à faire pour que ces techniques de communication deviennent pratiquement utilisables.

Dans un domaine en évolution aussi rapide, et où le nombre même des articles de synthèse, des colloques ou des livres consacrés au sujet croît exponentiellement [1-13], cette note ne peut avoir qu'un caractère schématique et la bibliographie n'être que partielle.

\section{Systèmes de télécommunications par fibres} optiques. - Comme tous les systèmes de transmission de l'information, ceux-ci sont analysables en termes de fonctions élémentaires : sources, modulateurs, milieux de transmission, amplificateurs, détecteurs, etc... (voir Fig. 1). Certaines de ces fonctions seront d'ailleurs avantageusement réalisables par les techniques de l'optique intégrée dont le but ultime (par analogie aux circuits intégrés électroniques) est la fabrication de circuits optiques complexes sous la forme d'éléments solides de petites dimensions : on espère obtenir ainsi soit des fonctions nouvelles, soit un gain en fiabilité ou une diminution du coût du système.

3. Milieu de transmission. - Nous nous bornerons ici aux fibres optiques. Par les pertes qu'elles introduisent et leur influence sur le bilan d'une liaison, elles posent évidemment un problème crucial, dont les méthodes de solution sont détaillées dans les références [14] à [17].

Le tableau I résume la classification de ces pertes.

\section{TABLEAU I}

\section{Classification des pertes}

Pertes dues AU MATÉRIAU INITIAL.

Absorption intrinsèque : Celle du matériau pur.

Absorption extrinsèque : Présence d'ions d'éléments de transition.

Présence de groupements hydroxyles.

Diffusion intrinsèque : Fluctuations figées. Fluctuations de composition.

Diffusion extrinsèque : Mauvaise élaboration du verre de départ (microfondus, dévitrifiés, etc...).

PERTES DUES AUX DÉFAUTS APPORTÉS PAR LE FIBRAGE.

Hétérogénéités d'indices par contraintes mécaniques. Imperfections géométriques.

Inclusions, bulles, etc...

Pertes dues a l'État de la fibre.

Influence de la courbure. 


\section{TABLEAU II}

Performances comparatives de divers types de fibres

\begin{tabular}{|c|c|c|c|c|c|c|}
\hline \multirow[t]{2}{*}{ Matériaux } & \multicolumn{2}{|c|}{$\begin{array}{c}\text { Pertes à } \\
\lambda=800 \mathrm{~nm} \\
(\mathrm{~dB} / \mathrm{km})\end{array}$} & \multirow{2}{*}{$\begin{array}{c}\text { Technique } \\
\text { de préparation }\end{array}$} & \multirow{2}{*}{$\begin{array}{l}\text { Ouverture } \\
\text { numérique }\end{array}$} & \multirow{2}{*}{$\begin{array}{l}\text { Diamètre cœur } \\
(\mu \mathrm{m})\end{array}$} & \multirow{2}{*}{$\begin{array}{l}\text { Observations } \\
\text { et références }\end{array}$} \\
\hline & Massif & Fibre & & & & \\
\hline $\begin{array}{c}\text { Verres } \\
\mathrm{Na}_{2} \mathrm{O}, \mathrm{CaO}, \mathrm{SiO}_{2}\end{array}$ & 50 & 56 & Barreau tube & 0,2 & 30 & {$[29]$} \\
\hline $\mathrm{Na}_{2} \mathrm{O}, \mathrm{PbO}, \mathrm{SiO}_{2}$ & $\begin{array}{l}20-25 \\
10-20\end{array}$ & $\begin{array}{c}35-50 \\
37\end{array}$ & $\begin{array}{l}\text { Barreau tube } \\
\text { Double creuset }\end{array}$ & $\begin{array}{l}0,5 \\
0,33\end{array}$ & 50 & {$\left[\begin{array}{l}{[23]} \\
{[19]}\end{array}\right.$} \\
\hline $\mathrm{Na}_{2} \mathrm{O}, \mathrm{B}_{2} \mathrm{O}_{3}, \mathrm{SiO}_{2}$ & $14-20$ & $20-25$ & Double-creuset & 0,15 & 10,20 & {$[23][28]$} \\
\hline $\mathrm{Na}_{2} \mathrm{O}, \mathrm{Al}_{2} \mathrm{O}_{3}, \mathrm{SiO}_{2}$ & $1 \mathrm{~dB}$ & & & & & [24] valeur estimée \\
\hline $\begin{array}{l}\text { Verre au lithium dopé } \\
\text { sodium }\end{array}$ & & 30 & Barreau-tube & 0,18 & $\varnothing$ gaine $=230$ & Fibre FORT multimode \\
\hline $\begin{array}{l}\text { Teneur élevée en silice } \\
(\mathrm{C}=\text { cœur } ; \mathrm{G}=\text { gaine }) \\
\mathrm{C}: \mathrm{Al}_{2} \mathrm{O}_{3}-\mathrm{SiO}_{2} \\
\mathrm{G}: \mathrm{SiO}_{2}\end{array}$ & & 20 & Hydrolyse à la flamme & 0,1 & $\varnothing$ gaine $=50$ & [30] Fibre monomode \\
\hline $\begin{array}{l}\mathrm{C}: \mathrm{P}_{2} \mathrm{O}_{5}-\mathrm{SiO}_{2} \\
\mathrm{G}: \mathrm{B}_{2} \mathrm{O}_{3}-\mathrm{SiO}_{2}\end{array}$ & & 2,7 & C. V. R. & 0,23 & 66 & $\begin{array}{l}\text { [31] Intérêt de } \mathrm{P}_{2} \mathrm{O}_{5} \text { pour } \\
\text { éliminer } \mathrm{OH}\end{array}$ \\
\hline $\begin{array}{l}\mathrm{C}: \mathrm{SiO}_{2} \\
\mathrm{G}: \mathrm{B}_{2} \mathrm{O}_{3}-\mathrm{SiO}_{2}\end{array}$ & & 1 à $1,06 \mu$ & $\begin{array}{l}\text { C. V. R. } \\
\text { Hydrolyse à la flamme }\end{array}$ & 0,17 & $\varnothing$ gaine $=50$ & {$[26][35]$} \\
\hline $\begin{aligned} \mathrm{C}: & \mathrm{B}_{2} \mathrm{O}_{3}-\mathrm{GeO}_{2-} \\
\mathrm{SiO}_{2} & \\
\mathrm{G}: & \mathrm{B}_{2} \mathrm{O}_{3}-\mathrm{SiO}_{2} \\
& \text { ou } \mathrm{SiO}_{2}\end{aligned}$ & & $\begin{array}{c}6 \\
2 \text { à } 1,06 \mu\end{array}$ & C. V. R. & $\begin{array}{c}0,23 \\
0,35 \\
\text { possible }\end{array}$ & 35 & {$[25]$} \\
\hline $\begin{array}{l}\text { Fibres spéciales } \\
\mathrm{SiO}_{2}\end{array}$ & & $4-8$ & & & 200 & $\begin{array}{l}\text { [32] Fibre Suprasil non gai- } \\
\text { née }\end{array}$ \\
\hline $\begin{array}{l}\text { Fibre ECOF excentrique } \\
\mathrm{SiO}_{2}-\mathrm{B}_{2} \mathrm{O}_{3}\end{array}$ & & 10 & Four à induction & & 100 au total & [33] Fibre NTT Japon \\
\hline Fibre Bell Labs $\mathrm{SiO}_{2}$ & & 11 & Plaque de $2,2 \mu$ & 0,14 & 22 & $\begin{array}{l}\text { Fibre spéciale à un seul } \\
\text { matériau [32] }\end{array}$ \\
\hline
\end{tabular}

Remarques: 1. C. V. R. : réaction chimique en phase vapeur.

2. Ouverture numérique $\mathrm{ON}=\left(n_{\mathrm{C}}^{2}-n_{\mathrm{G}}^{2}\right)^{\frac{1}{2}}$.

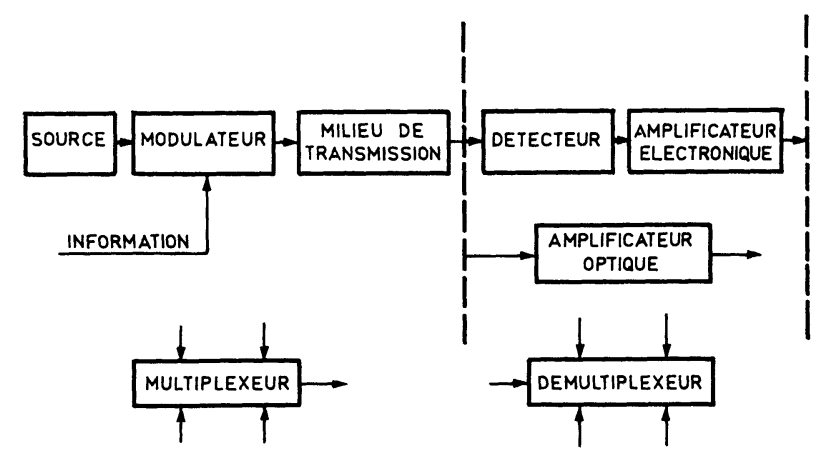

FIG. 1. - Schéma d'une transmission d'information par voie optique.

Le problème de la pureté des produits chimiques de départ et de la propreté de leur fusion est particulièrement dramatique pour les verres classiques (qui ne le sont en fait pas). Il est partiellement résolu actuelle- ment pour certains d'entre eux [18-20]. L'utilisation de matériaux à teneur élevée en silice, avec des technologies voisines de celles des composants à semiconducteurs (déposition en phase vapeur, etc...) permet une approche moins pénible et conduit actuellement aux meilleurs résultats ; cependant le fibrage doit se faire à température plus élevée avec les risques de pollution correspondants.

L'importance relative des mécanismes intrinsèques d'absorption et de diffusion [34] pourrait faire que d'autres composés que la silice et la silice dopée soient finalement choisis : récemment Pinnow [24] a montré que certaines compositions du système $\mathrm{Na}_{2} \mathrm{O}-\mathrm{Al}_{2} \mathrm{O}_{3}$ $\mathrm{SiO}_{2}$ présentaient moins de diffusion intrinsèque et probablement moins d'absorption que la silice pure. Le tableau II résume les performances des fibres les meilleures obtenues jusqu'à maintenant. Les mesures de diffusion et d'absorption par microcalorimétrie sont traitées dans les références [21, 22]. 
L'étude fondamentale de l'absorption intrinsèque de presque tous les composés reste à faire : la structure même de verres relativement simples est encore controversée : des techniques comme la résonance de spin [39] ou la diffusion de neutrons [41] peuvent contribuer à leur étude. Les centres colorés créés par le fibrage [40] ou l'influence de rayonnements divers commencent à être étudiés pour déterminer la durée de vie des liaisons.

Les effets non linéaires dans les fibres : Brillouin [38], Raman [36, 37] sont également l'objet de recherches, tant sur le plan fondamental que par les limitations à la puissance transportée qu'ils entraînent.

Les problèmes de tenue mécanique des fibres, de leur gainage [73, 74] (qui peut améliorer notablement celle-ci) du câblage d'ensembles de fibres ainsi que les problèmes de raccordement et de connecteurs devront recevoir des solutions transposables à l'échelle industrielle pour que les systèmes correspondants puissent quitter le laboratoire.

4. Sources et détecteurs. - Les détecteurs classiques, du moins jusqu'à un micron, ne posent pas de problèmes particuliers. Il n'en va pas de même pour les sources. Pour être compatibles avec les fibres, cellesci doivent présenter les propriétés suivantes :

- puissance émise suffisante,

- rendement élevé (puissance d'alimentation faible),

- longue durée de vie,

- émission à une longueur d'onde où l'absorption des fibres est faible et les détecteurs efficaces.

Les systèmes actuellement les plus étudiés sont basés sur les dispositifs à double hétérostructure dans le système $\mathrm{Al}_{x} \mathrm{Ga}_{1-x} \mathrm{As}$ : diodes électroluminescentes ou lasers [42-43]. La physique de leurs défaillances commence à être maîtrisée [45]. La longueur d'onde au voisinage de $1,06 \mu$ présente également beaucoup d'intérêt. Pour cela, le système $\operatorname{In}_{x} \mathrm{Ga}_{1-x}$ As fait également l'objet de nombreux travaux, tant pour les émetteurs que pour les récepteurs [46]. L'épitaxie par jet moléculaire [47] et l'utilisation de réseaux de couplage distribués $[48,59]$ se révéleront sans doute des technologies intéressantes, la dernière permettant une meilleure sélection des modes et un seuil d'oscillations plus bas.

Les lasers miniatures (à pompage par diode électroluminescente) à base de matériaux au néodyme sont également intéressants [44] $\left(^{1}\right)$. Ils nécessitent cependant un modulateur séparé $[67,72]$. Parmi les matériaux nouveaux, l'ultraphosphate de néodyme : $\mathrm{NdP}_{5} \mathrm{O}_{14}$ et ses solutions solides avec le lanthane est le plus étudié [49] $\left({ }^{2}\right)$, mais d'autres tels $\mathrm{LiNdP}_{4} \mathrm{O}_{12}$ [50] seront peut-être utilisables. Ces matériaux ont des durées de vie radiative de l'ordre de $100 \mu$ s pour une

(1) Noblanc, J. P., Communication privée.

(2) Noblanc, J. P., Budin, D., Marais, M., Communication privée.

REVUE DE PHYSIQUE APPLIQUÉE. - T. 10, № 6, NOVEMBE 1975 concentration en néodyme élevée, ce qui entraîne une possibilité de pompage efficace et un faible seuil laser pour des échantillons de petites dimensions.

5. Amplificateurs. - L'importance de cette fonction dans les transmissions optiques n'est pas encore évidente. Deux approches semblent $a$ priori possibles : celle des fibres amplificatrices (dopées au néodyme) [52] ou celle, plus probable, qui utilise des structures analogues aux lasers à semi-conducteurs [51].

6. Optique intégrée. - Bien que des progrès particulièrement rapides et spectaculaires aient déjà été réalisés, d'autres sont encore nécessaires pour que tout le potentiel de cette technique s'accomplisse. Une grande partie de ces efforts de recherche en optique intégrée a porté sur l'essai de matériaux nouveaux [53, 54,61 ], mais la situation n'est pas totalement satisfaisante. Certes, des guides ayant des pertes très faibles $(0,05 \mathrm{~dB} / \mathrm{cm})$ ont été réalisés dans des verres et des couches organiques, mais les modulateurs et les commutateurs ne peuvent être réalisés dans ces matériaux. Les matériaux ferroélectriques comme le niobate et le tantalate de lithium sont particulièrement étudiés du fait de leurs grands coefficients électro-optiques [53, $56,57,67]$ mais les sources n'y sont pas réalisables. Les guides, les modulateurs, les sources à injection, les détecteurs à diode p.n ou Schottky peuvent être fabriqués relativement aisément dans les composés III-V [6164] mais la limitation latérale des guides doit encore faire l'objet d'études ; de plus les pertes de ces matériaux sont généralement assez élevées et la résistivité parfois trop faible pour permettre la réalisation de certains types de modulateurs ou de commutateurs. Cependant, l'intégration monolithique de fonctions actives et passives dans GaAs a déjà été démontrée [63]. Mais il faut noter que pour pouvoir tirer tout le bénéfice des techniques de multiplexage en fréquence, des ensembles de sources à fréquences différentes devront être réalisés sur le même substrat.

En ce qui concerne les composés II-VI, les techniques classiques des semi-conducteurs leur sont applicables, mais il est impossible de réaliser des jonctions p.n de qualité suffisante dans ces matériaux.

Finalement, la réponse sera peut-être dans une circuiterie hybride où les matériaux seront combinés par hétéroépitaxie ou par diverses techniques de déposition.

Les matériaux magnétiques [53, 69-72] sont également envisageables. Ils sont les seuls à permettre la réalisation de dispositifs non réciproques. Des guides et des modulateurs ont déjà été réalisés dans des couches minces de grenats, cependant les imperfections des épitaxies limitent sans doute les vitesses de modulation possibles et ne permettent pas encore la réalisation de sources laser fonctionnant en continu.

Des techniques particulières, permettant des résolutions élevées, sont nécessaires pour la réalisation des guides. Le masquage électronique a été utilisé avec un succès certain [54] mais des études de résines sont 
encore nécessaires. Les techniques holographiques et la gravure ionique permettent la réalisation de réseaux à pas très fins $[53,59,61]$. Le bombardement du substrat par des faisceaux focalisés d'ions ou de protons pourrait également être une technique envisageable. Les défauts d'implantation ou les effets de dopage chimique par les impuretés implantées ont déjà été utilisés pour la réalisation de guides plans $[55,61,62]$. Les problèmes de couplage [58] sont également loin d'être triviaux : les applications les plus intéressantes de l'optique intégrée nécessiteront le couplage efficace entre des fibres optiques monomodes et des circuits optiques intégrés, couplage qui devra être réalisé avec des tolérances dimensionnelles faibles. A l'autre extrémité de la chaîne, il serait intéressant que les interfaces entre circuits optiques et électroniques réalisés sur le même substrat soient optimisés, ce qui n'est pas le cas actuellement.

7. Conclusion. - L'utilisation pratique de systèmes de télécommunications par fibres optiques est maintenant pratiquement certaine. Le futur de l'optique intégrée reste plus problématique. Les progrès rapides réalisés en quelques années n'ont pu l'être que par la conjonction d'effets multidisciplinaires dont nous avons essayé de faire un point rapide.

\section{Bibliographie}

\section{Articles de revue.}

\subsection{TÉLÉCOMMUNICATIONS OPTIQUES.}

[1] Maurer, R. D., Proc. IEEE 61 (1973) 452.

[2] Miller, S. E., Marcatili, E. A., TI, L.-G., Proc. IEEE 61 (1973) 1703.

[3] Miller, S. E., Li, T.-G., Marcatili, E. A., Proc. IEEE 61 (1973) 1726

[4] Balkanski, M. et Lallemand, P., Photonics Comptes Rendus d'un Colloque D. G. R. S. T. (Gauthier-Villars) 1974.

[5] Cook, J. S., Recherche 3 (1972) 426.

[6] Ann. Télécommun. 29 (1974) N 5-6 (1974) Numéro spécial consacré aux télécommunications par fibres optiques.

[7] Rev. Tech. THOMSON-CSF 6 (déc. 1974) $\mathrm{N}^{\circ} 4$. Numéro spécial consacré à l'optique intégrée et aux télécommunications par fibres optiques.

\subsection{OPTIQUE INTÉGRÉE.}

[8] TAYLOR, H. F., YARIV, A., Guided wave optics, Proc. IEEE 62 (1974) 1044.

[9] Chang, W. S., Muller, M. W., Rosenbaum, F. J., Integrated Optics dans Laser Appl. 2 (Academic Press N. Y.) 1974.

[10] Technical Digest of Topical Meeting on Optical Fiber Transmission, Williamsburg (Virginie) 1975.

[11] IEEE Trans. Microwave Theory Tech. MTT 23 (1975) Numéro spécial consacré à l'optique intégrée.

[12] Bulabois, J. (Association pour l'Etude de Problèmes avancés) Optique Intégrée C. R. de la convention D. R. M. E. $\mathrm{N}^{\circ} 383 / 701974$.

[13] Comptes Rendus du Colloque sur l'optique des ondes guidées Paris 1975.

\section{Matériaux pour fibres optiques et réalisation des} fibres.

[14] ReYmond, J. C., réf. [7] p. 899.

[15] ERNEST, J., réf. [4] p. 377.

[16] Passaret, M., réf. [6] p. 179.

[17] Haussonne, J. M., Bull. Soc. Fr. Céram. 105 (1974) 35.

[18] Zief, M., Horvath, J., Theodorou, N., réf. [10]. Article Tu B3.

[19] Faulstich, M., Krause, D., Neuroth, N., Reit Mayer, F., réf. [10] Article Tu B5.

[20] Gossink, R. G., van Ass, H. M., réf. [10] Article Tu B4.

[21] Broquet, I., Thèse de $3^{\mathbf{e}}$ cycle, Faculté des Sciences d'Orsay (1973).
[22] Zaganiaris, A., Appl. Phys. Lett. 25 (1974) 345 et Thèse de $3^{\mathrm{e}}$ cycle. Université de Rennes (1974).

[23] Newns, G. R., réf. [10] Article Tu B1.

[24] PInNow, D. A. et coll., Mater. Res. Bull. 10 (1975) 133. Voir aussi : mêmes auteurs : réf. [10] Article Tu A3.

[25] Mac Chesnay, J. B., O’Connor, P. B., Presby, H. M., Proc. IEEE 62 (1974) 1280.

[26] TASker, G. W., French, W. G., Proc. IEEE 62 (1974) 1281. Voir aussi : mêmes auteurs : réf. [10] Article Tu A2.

[27] Van Uitert, L. G. et coll., Mater. Res. Bull. 8 (1973) 469.

[28] Newns, G. R. et coll., Electron. Lett. 10 (1974) 201.

[29] Stewart, C. E. E., BLACK, P. N., Electron. Lett. 10 (1974) 53.

[30] Kobayashi, S. et coll., Electron. Lett. 10 (1974) 410.

[31] Payne, D. N., Gambling, W. A., Opt. Commun. 13 (1975) 422.

[32] Katser, P., Astle, H. W., Bell Syst. Tech. 53 (1974) 1021.

[33] Miyashita, N. et coll., J. Appl. Phys. 45 (1974) 808.

[34] Pinnow, D. A. et coll., Aprl. Phys. Lett. 22 (1973) 527.

[35] DabBy, F. W. et coll., Mater. Res. Bull. 10 (1975) 425.

[36] Walrafen, G. E., J. Chem. Phys. 62 (1975).

[37] Walrafen, G. E., Appl. Spectrosc. 29 (1975) 179.

[38] Stone, J., Appl. Opt. 12 (1973) 1824.

[39] Калчмомa, S. S. et coll., Phys. Status Solidi B 68 (1975) K 137.

[40] Kaiser, P., J. Opt. Soc. Am. 64 (1974) 475.

[41] Loshmanov, A. A. et coll., Sov. Phys. Cristallogr. 19 (1974) 168.

\section{Sources et détecteurs.}

[42] KRessel, H., réf. [4] p. 1.

[43] Lebailly, J., réf. [4] p. 27.

[44] Barnes, N. P., J. Appl. Phys. 44 (1973) 230.

[45] Deloach, B. C., Recent Advances in Semiconductor lasers : the Physics of Failure of GaAs Solid State Lasers. Article I.1, VIII Internat. Quantum. Electr. Conf. San Francisco, 1974.

[46] Nuese, C. J., Enstrom, R., IEEE Trans. Electron Devices ED-19 (1972) 1067

[47] Сho, A. Y., Casey, H. C., Appl. Phys. Lett. 25 (1974) 288.

[48] Burnham, R. D., Scifres, D. R., Streifer, W., Appl. Phys. Lett. 26 (1975) 644.

[49] Weber, H. P., Damen, T. C. et coll., Appl. Phys. Lett. 22 (1973) 534

[50] Chinn, S. R., Hong, H. Y. P., Appl. Phys. Lett. 26 (1975) 649.

\section{Amplificateurs.}

[51] Zeidler, G., SchicketanZ, D., Siemens Forsch. 4 (1973) 227.

[52] Koester, C. J., Snitzer, E., Appl. Opt. 3 (1964) 1182. 


\section{Optique intégrée.}

\subsection{GUIDES DIVERS.}

[53] Kogelnik, H., réf. [11] p. 1.

[54] Ostrowsky, D. B., Roy, A. M., réf. [7] p. 955.

[55] Rao, E. V. K., Moutonnet, D., J. Appl. Phys. 46 (1975) 955.

[56] ChIn, G. Y. et coll., Appl. Phys. Lett. 26 (1975) 637.

[57] ShaH, M. L., Appl. Phys. Lett. 26 (1975) 652.

\subsection{Couplage.}

[58] Kogelnik, H., réf. [4] p. 247.

\section{3 LASERS DISTRIBUÉS.}

[59] SHYH WANG, Wave Electronics 1 (1974-75) 31.

[60] Ostrowsky, D. B., SeVin, J., réf. [4] p. 309.

5.4 OPTIQUE INTÉGRÉE DANS LES COMPOSÉS III-V.

[61] Evtuhov, V., Yariv, A., réf. [11] p. 44.

[62] FAVENNEC, P. N., RAO, E. V. K., Ion Implantation in Semiconductors and other materials (S. Namba Ed., Plenum Press, N. Y.) 1975.

[63] Reinhart, F. K., Logan, R. A., Appl. Phys. Lett. 26 (1975) 516.

[64] Campbell, J. C., Blum, F. A., Shaw, D. W., Appl. Phys. Lett. 26 (1975) 640.

\subsection{DÉTECTEURS INTÉGRÉS.}

[65] Ostrowsky, D. B. et coll., Appl. Phys. Lett. 22 (1973) 463. [66] Cheng, Chung Tseng, Shyh Wang, Appl. Phys. Lett. 26 (1975) 632.

\subsection{ElECTRO-OPTIQUE.}

[67] Kaminow, I. P., réf. [11] p. 57 et réf. [4] p. 267.

\subsection{ACOUSTO-OPTIQUE.}

[68] Harteman, P., réf. [7] p. 1091.

\subsection{MAGNÉTO-OPTIQUE.}

[69] Wemple, S. H. et coll., Appl. Phys. Lett. 22 (1973) 331. [70] Daval, J. et coll., Conference Intermag (Londres) 1975, à paraître dans IEEE Trans. Magn., 1975.

[71] Hepner, G. et Desormiere, B., réf. [7] p. 1115 et réf. [4]. p. 155.

[72] Legall, H., réf. [4] p. 123.

6. Fibres gainées plastiques.

[73] Kaiser, P., Hart, Ac., Blyler, L. L., Appl. Opt. 14 (1975) 156.

[74] TANAKA, S. et coll., Electron. Lett. 11 (1975) 153. 